\title{
Absence of an Autonomic Sign Assists in the Diagnosis of Extratemporal Lobe Epilepsy Manifesting Generalized Convulsion with Retained Awareness
}

\author{
Shuichiro Neshige ${ }^{1,2}$, Riki Matsumoto ${ }^{1}$, Morito Inouchi ${ }^{1}$, Katsuya Kobayashi ${ }^{1}$, \\ Akihiro Shimotake ${ }^{3}$, Hirofumi Maruyama ${ }^{2}$, Ryosuke Takahashi ${ }^{1}$ and Akio Ikeda ${ }^{3}$
}

\begin{abstract}
:
We herein report two epilepsy patients with the seizure focus in the non-dominant hemisphere manifesting secondarily generalized convulsion (sGC) with retained awareness characterized by a lack of autonomic signs although GC was complicated by respiratory arrest. Given the semiology and electrophysiological findings, the seizure activity was considered to propagate to the supplementary-motor area and the bilateral primarymotor area, with a clinical manifestation of sGC. The absence of autonomic signs during GC can be a key indicator that the seizure remains in the bilateral suprasylvian area and thus does not involve the region necessary for awareness preservation, which may assist in the diagnosis of this atypical epileptic seizure.
\end{abstract}

Key words: generalized, consciousness, autonomic symptoms, non-dominant hemisphere, insula

(Intern Med 58: 1151-1155, 2019)

(DOI: 10.2169/internalmedicine.1740-18)

\section{Introduction}

Focal seizures occasionally develop to secondarily generalized convulsion (sGC), which presents as sustained-tonic limb extension spreading from the axial muscles to the limbs and subsequent rhythmic clonic jerking with a loss of awareness (LOA) and deep unresponsiveness (1). Although patients usually lose awareness during sGC, a few cases with retained awareness have been reported (2-4). Because the clinical features of this unusual seizure have remained largely unclear, this rare population may be at risk of a misdiagnosis of psychogenic non-epileptic seizure (PNES) (2).

We herein report two epilepsy patients manifesting sGC with retained awareness whose semiology was characterized by the absence of autonomic signs during sGC, although the seizures were complicated by respiratory arrest. Autonomic signs, which include cutaneous, cardiovascular, gastrointestinal and respiratory manifestations (5), can be of diagnostic value as localizing signs (e.g., insula, amygdala, operculum regions) $(6,7)$. Since sGC commonly manifests with autonomic signs (8), we considered this atypical semiology namely the absence of autonomic signs - to be unique to sGC with preserved awareness as opposed to that with impaired awareness. Although "sGC" is currently not reccomended as a diagnosis, we use this term to describe this atypical seizure, which is unclassifiable according to the International League Against Epilepsy (ILAE) classification (9).

\section{Case Reports}

\section{Case 1}

A right-handed 17-year-old boy was admitted to our hospital for the evaluation of refractory epileptic seizures. His seizure history had started at 15 years of age with 2 GC episodes. After starting antiepileptic drug (AED) treatment, his seizures would begin with an uncharacteristic feeling, such as dizziness, followed by motion arrest and left side-

\footnotetext{
${ }^{1}$ Department of Neurology, Kyoto University Graduate School of Medicine, Japan, ${ }^{2}$ Department of Clinical Neuroscience and Therapeutics, Hiroshima University Graduate School of Biomedical and Health Sciences, Japan and ${ }^{3}$ Department of Epilepsy, Movement Disorders and Physiology, Kyoto University Graduate School of Medicine, Japan

Received: June 26, 2018; Accepted: August 31, 2018; Advance Publication by J-STAGE: December 18, 2018

Correspondence to Dr. Riki Matsumoto, matsumot@kuhp.kyoto-u.ac.jp
} 

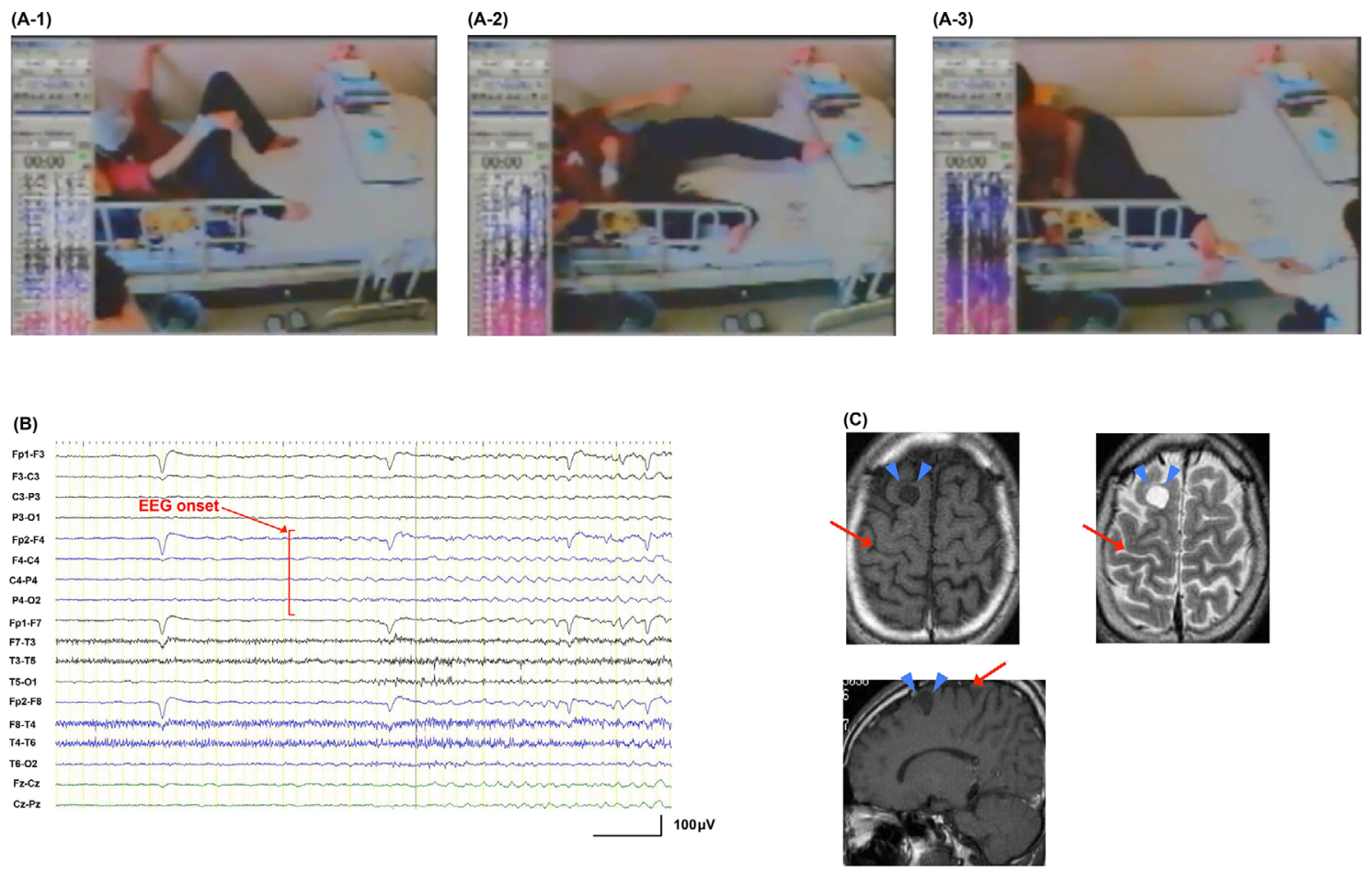

Figure 1. Semiology, EEG, and MRI findings in Case 1. (A) Photographs showing asymmetric tonic posture (A-1) followed by Figure 4 sign (A-2) and subsequent left side-dominant bilateral clonic movements (A-3). (B) EEG shows ictal activities arising from the right frontal region. (C) Brain MRI of axial T1WI (left upper), axial T2WI (right upper) and sagittal T1WI (left lower) shows cystic lesions (blue arrowheads) in the right superior frontal gyrus that are anatomically located anterior to the supplemental motor area. Red arrows indicate the location of the central sulcus. Informed consent was obtained from the patient for the publication of part (A) of this figure.

dominated generalized-tonic posture. Even under dual-AED treatment consisting of carbamazepine $(600 \mathrm{mg} /$ day $)$ and zonisamide (300 mg/day), the seizures occurred every week. Electroencephalography (EEG) revealed regional sharp waves and intermittent irregular $4-5 \mathrm{~Hz}$ slow activity in the right frontal region.

A two-week period of video-EEG monitoring captured three clinical habitual seizures, each consisting of an initial floating or rising sensation that was not clearly localized within the body followed by a sensation of gradually laying down on the bed accompanied by asymmetric-tonic posture (left-dominant bilateral shoulder and hip joint abduction) (Fig. 1A-1) and subsequent left hand convulsions to make a "Figure 4 Sign" (Fig. 1A-2). The seizures finally developed to sGC (bilateral left-side dominant asymmetric generalized clonic jerks including head version to the left). Because of these generalized clonic movements, the patient would hit his extended extremities on the bed rail (Fig. 1A-3). Of note, there were no autonomic signs, such as drooling, excessive salivation, nasal secretion, or profuse sweating at any time throughout his seizure including the GC, which was complicated by respiratory arrest. During some episodes, nurses presented images and asked him to remember sets of random words; during the GC, he was totally unresponsive to these stimuli, but after the seizure ended he could immediately recall the images and words.
Ictal EEG showed low-voltage, 5-Hz rhythmic theta activity in the right frontal region lasting for 3-4 seconds (Fig. 1B), which preceded the clinical onset by a few seconds, followed by high-voltage, 6-Hz rhythmic theta activity in the right-dominant bilateral frontal regions. Once the seizure became generalized, the EEG was obscured by electromyography artifacts (as shown in the left side of Fig. 1A-3). Although the waveforms were limited in the movement artifacts, electrocardiograms (ECGs) did not show any evident cardiogenic changes. An invasive evaluation revealed the epileptic focus at and surrounding a dysembrionic neuroepithelial tumor in the right superior frontal gyrus located rostral to the supplementary-motor area (SMA) (Fig. 1C). He remained seizure-free for six years following resection of the focus at and around the tumor. Our examination of the epicortical recording of ictal EEG in this patient has already been published elsewhere for entirely different purposes (10).

\section{Case 2}

A right-handed 58-year-old woman with refractory epilepsy was admitted for a pre-surgical evaluation. Her initial seizure had been a nocturnal GC with LOA at age 15. She reported suffering from simple partial seizures (SPSs) characterized by several minutes of paresthesia in her left leg from the age of 35 . Once an SPS started, it occurred repeti- 

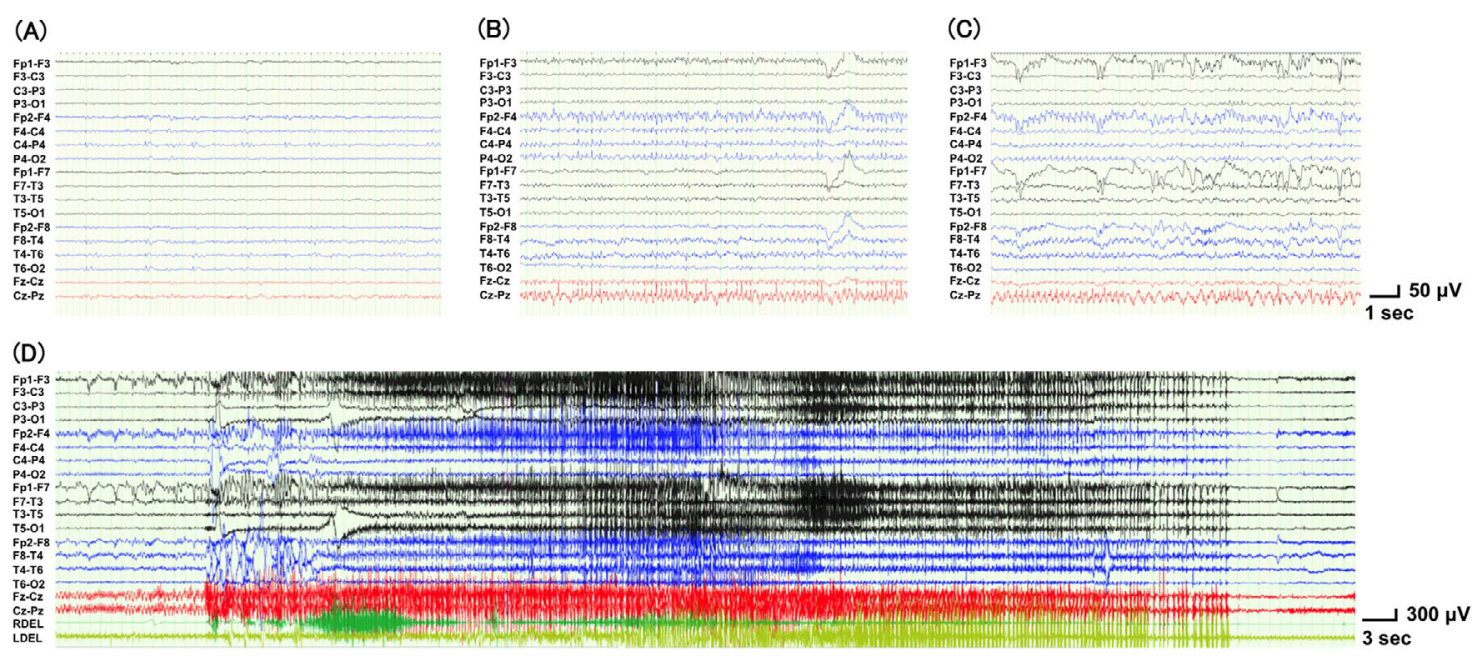

(E)

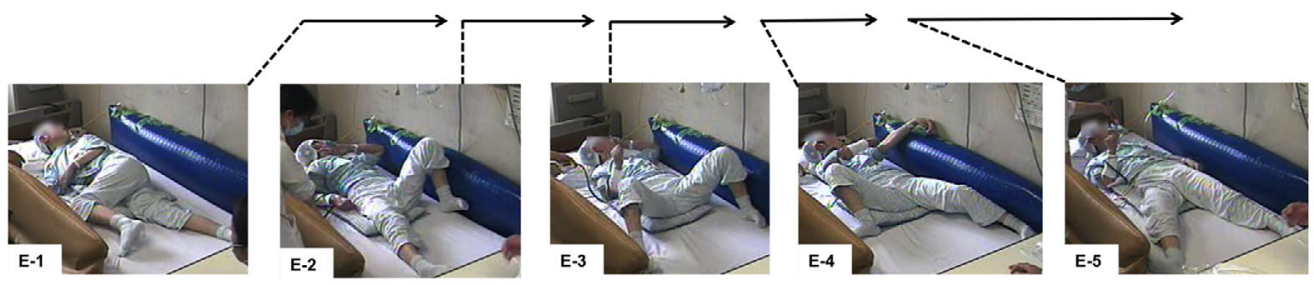

(F)

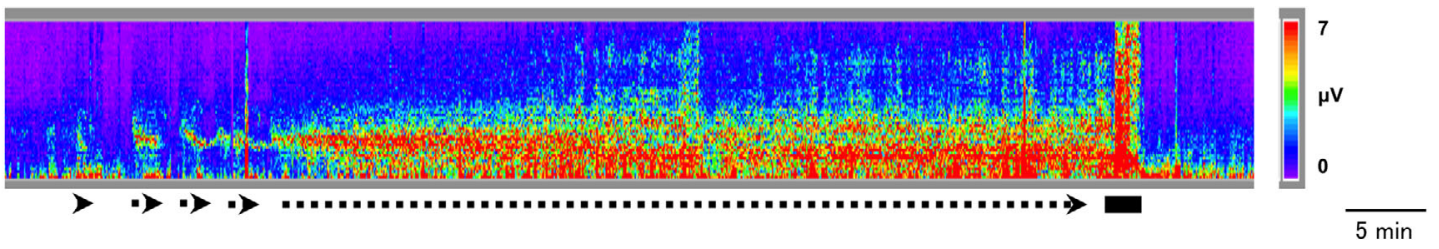

Figure 2. EEGs and semiology findings in Case 2. EEGs were recorded with a sampling rate of 500 $\mathrm{Hz}$ and time constant of 10 seconds using shallow cup electrodes according to the International 10-20 System. Interictal EEG (A); ictal EEG during simple partial seizure (SPS) cluster (B and C); ictal EEG developing to generalized convulsion and clinical semiology with head version to the left, asymmetric-tonic seizure, Figure 4 Sign, and generalized clonic seizure (D and E); and a digital spectrum assay (DSA) analyzed from the data of C4, F4, and P4 electrodes (F). The DSA over an 80-minute period is shown. The dotted lines with arrowheads at the bottom of the DSA indicate SPS and SPScluster, and the bar indicates the time during secondary generalized convulsion. Due to the patient's movements, the recording quality of the electromyograms of the right deltoid (DEL) muscle is poor (as shown in the latter part of Fig. 1D). Informed consent was obtained from the patient for the publication of part $\mathrm{E}$ of this figure.

tively in several 10-minute cycles (SPS-cluster) followed by convulsion of the left extremities and subsequent sGC. She reported being aware throughout the seizures, including the generalized phase. Given her unusual assertion that she maintained consciousness during the GC, her primary doctor concluded that she had both epileptic seizures and PNES. Although she was treated with multiple AEDs at once, including phenytoin (150 mg/day), valproic acid (600 mg/day) and zonisamide (50 mg/day), her seizures remained refractory to treatment.

Brain magnetic resonance imaging (MRI) and fluorodeoxyglucose-positron emission tomography revealed mild atrophy and mildly reduced glucose metabolism, respectively, in the bilateral centroparietal area. EEG revealed regional right frontocentral and centrotemporal spikes (Fig. 2A).

Video-EEG monitoring captured 12 habitual seizures. Ictal EEG showed rhythmic theta on the right frontocentral and semirhythmic theta in the right midtemporal region followed by repetitive spikes (Fig. 2B and C). Once a seizure started, the SPS occurred every 10 to 30 minutes (SPS-cluster). On some occasions, her SPS developed to sGC characterized by head version to the left accompanied by asymmetric-tonic posture with bilateral-limb abduction and extension of the right limbs to make a "Fig. 4 Sign," followed by asynchronous bilateral clonic jerks of the extremities (Fig. 2E). Although the GC was complicated by respiratory arrest for several 10-second periods, notably, she did not exhibit any 
autonomic signs throughout the seizure. EEG revealed rhythmic activity on the right frontocentrotemporal region that preceded the onset of the asymmetric-tonic posture (Fig. 2D). Ictal cardiogenic changes were not clearly visible in ECGs. During a seizure, she could not reply to people around her but was aware of what was happening to her. To assess her awareness and memory, we administered a simple test battery during a GC, including verbal questions and the presentation of random words which the patient was asked to remember and recall. When the seizure ended, she was able to immediately reply to the questions and could also recall the random words. The step-wise development of her seizure (from tandem SPS representations to SPS-cluster and subsequent sGC) was clearly illustrated by a digital spectrum assay (Fig. 2F).

\section{Discussion}

We herein report two cases of $\mathrm{sGC}$ with retained awareness whose semiology suggests important issues related to the diagnosis of this atypical seizure. First, the semiology during sGC in our cases was slightly different from those of typical GC with LOA in that there were no autonomic signs, such as drooling and excessive salivation, even though the seizures were accompanied by respiratory arrest. Second, asymmetric-tonic seizures prior to clonic seizures were more evident in these cases than in typical GC with LOA. This is the first report to highlight these semiological findings, which may assist in the diagnosis of sGC with retained awareness.

In Case 1, given the findings of semiology, EEG and MRI, the seizures presumably originated from the right SMA and propagated to the bilateral primary-motor area (M 1) falling into sGC. In both cases, the step-wise evolution in their semiology and ictal-EEG findings suggested that the seizures were not PNES but rather were consistent with sGC (11). The semiology and ictal EEG findings in Case 2 indicated that the seizure activity originated from the right centroparietal region and involved the right SMA, leading to asymmetric-tonic posture, with seizure activation finally increasing in the bilateral M1, and developing to sGC.

SMA seizure should be discussed with regard to our cases (12). The middle and initial segments of the seizures in cases 1 and 2, respectively, were consistent with SMA seizure, whereas the latter segments of the seizures in both cases progressed to $\mathrm{sGC}$, which is prominent in clonic rather than tonic seizure. Based on the results of a stimulation study, we speculated that the latter segments of the seizures were primarily generated by epileptic activity on the M1 rather than on the SMA (13). If the epileptic activity involves the bilateral motor pathways by spreading through the corpus callosum (2) or if the epileptic seizures spread to the bilateral M1 independently, seizures can clinically represent sGC even when the whole brain is not involved in the seizure. We thus consider that the present cases of sGC reflect seizure activation in the bilateral motor areas including the M1 and SMA, but not necessarily in the whole brain. However, this is still a hypothesis rather than a conclusion, as the bilateral ictal discharges were not shown in our EEGs due to motion artifacts in both cases.

One possible mechanism underlying sGC with retained awareness is that a region that is necessary for maintaining consciousness, such as the subcortical arousal system (the thalamus and upper brainstem) or the limbic system (14), is spared from seizure propagation even though the seizure activity is clinically "generalized." This hypothesis is supported by the absence of autonomic signs in our cases, as autonomic signs are potential localizing signs for the cortical regions responsible for the autonomic system (i.e., limbic structure) based on an ictal EEG study (15) and a cortical stimulation study (16). Autonomic signs, particularly drooling/foaming resulting from a lack of swallowing, profuse sweating and excessive salivation, are commonly prominent in secondarily generalized tonic clonic seizures (8); in sGC, however, the absence of autonomic signs is acceptable because an electrocorticography study has suggested that some regions can be spared to some degree, even if a seizure is clinically generalized (17). Given previous suggestions that generalized seizures are not truly generalized $(18,19)$, it is acceptable that the semiology of sGC is clinically heterogeneous and lacks autonomic signs. Therefore, we considered that the absence of autonomic signs during sGC could be an important semiology associated with this specific situation (i.e., the sparing of an area necessary for maintaining consciousness from the seizure activities).

Commonalities between the present two cases and four previously reported cases (2-4) should be acknowledged. First, all six of these cases were diagnosed as extratemporal lobe epilepsy and showed asymmetric or symmetric-tonic posture prior to GC. This commonality suggests that epileptic seizure arising from the suprasylvian region finally remained in the bilateral M1, representing a GC, without propagating to the limbic structures. The absence of autonomic signs supports this speculation (there were no descriptions of autonomic signs during GC in the four previous reports). Second, the seizure focus was documented in the non-dominant hemisphere (right hemisphere) in five of these six cases. Preponderance in the non-dominant hemisphere would enable the sparing of regions involved in the processing of language and memories and thus likely contributes to this semiology.

Some limitations associated with the present study include the small number of evaluated patients, the lack of ictal EEG and ECG findings during clinically generalized seizures and the limited diagnostic accuracy of the use of clinical signs in the identification of intracerebral symptomatic regions, as the determination of propagation of seizure activity in particular brain regions based on semiology is largely speculative.

In conclusion, clinicians must be aware that sGC without LOA can be found in focal epilepsy, particularly in patients with an extratemporal seizure focus in the non-dominant 
hemisphere. The absence of autonomic signs may be an important semiology for the diagnosis of this unique seizure. Understanding this finding might help avoid a misdiagnosis of PNES. The further accumulation of cases is expected to confirm the clinical utility of this finding, along with that of the focus existing in the non-dominant hemisphere.

\section{Author's disclosure of potential Conflicts of Interest (COI).}

Akihiro Shimotake and Akio Ikeda are current members (Riki Matsumoto and Morito Inouchi are previous members) of the Department of Epilepsy, Movement Disorders and Physiology, which was an endowment department supported by a grant from Eisai Pharmaceutical Co, NIHON KOHDEN CORPORATION, Otsuka Pharmaceutical Co., and UCB Japan Co., Ltd., or Eisai Pharmaceutical Co, until May 2018.

\section{Financial Support}

This work was partly funded by the Japan Ministry of Education, Culture, Sports, Science and Technology (MEXT) KAKENHI Grant Numbers 15H05874, 17H05907, and Japan Society for the Promotion of Science (JSPS) KAKENHI Grant Numbers 26293209.

\section{Acknowledgement}

We thank our patients for agreeing to share their information in this publication. Written informed consent to publish this case report was obtained from both of the patients.

\section{References}

1. Goldensohn ES, Glaser GH, MA G. Merritt's Textbook of neurology. 7th ed. Lea and Febiger, Philadelphia, 1984: 635-636.

2. Bell WL, Walczak TS, Shin C, Radtke RA. Painful generalised clonic and tonic-clonic seizures with retained consciousness. J Neurol Neurosurg Psychiatry 63: 792-795, 1997.

3. Weinberger J, Lusins J. Simultaneous bilateral focal seizures without loss of consciousness. Mt Sinai J Med 40: 693-696, 1973.

4. Botez MI, Serbănescu T, Stoica I. The problem of focal epileptic seizures on both parts of the body without loss of consciousness. Psychiatr Neurol Neurochir 69: 431-437, 1966.

5. Baumgartner C, Lurger S, Leutmezer F. Autonomic symptoms during epileptic seizures. Epileptic Disord 3: 103-116, 2001.
6. Freeman R, Schachter SC. Autonomic epilepsy. Semin Neurol 15: 158-166, 1995.

7. Burgess R. Autonomic signs associated with seizures. In: Epileptic Seizures. Pathophysiology and Clinical Semiology. Lüders HO, Ed. Churchill Livingstone, New York, 2000: 631-641.

8. Epilepsy: A Comprehensive Textbook. Engel J Jr, Pedly TA, Eds. Lippincott-Raven Publishers, Philadelphia, 1997: 567-577.

9. Fisher RS, Cross JH, D'Souza C, et al. Instruction manual for the ILAE 2017 operational classification of seizure types. Epilepsia 58: 531-542, 2017.

10. Ikeda A, Hirasawa K, Kinoshita M, et al. Negative motor seizure arising from the negative motor area: is it ictal apraxia? Epilepsia 50: 2072-2084, 2009.

11. Theodore WH, Porter RJ, Albert $\mathrm{P}$, et al. The secondarily generalized tonic-clonic seizure: a videotape analysis. Neurology 44: 1403-1407, 1994.

12. Ikeda A, Sato T, Ohara S, et al. "Supplementary motor area (SMA) seizure" rather than "SMA epilepsy" in optimal surgical candidates: a document of subdural mapping. J Neurol Sci 202: 43-52, 2002.

13. Hamer HM, Lüders HO, Rosenow F, Najm I. Focal clonus elicited by electrical stimulation of the motor cortex in humans. Epilepsy Res 51: 155-166, 2002.

14. Blumenfeld H. Impaired consciousness in epilepsy. Lancet Neurol 11: 814-826, 2012.

15. Baumgartner $C$, Gröppel $G$, Leutmezer F, et al. Ictal urinary urge indicates seizure onset in the nondominant temporal lobe. Neurology 55: 432-434, 2000.

16. Oppenheimer SM, Gelb A, Girvin JP, Hachinski VC. Cardiovascular effects of human insular cortex stimulation. Neurology 42: 1727-1732, 1992.

17. Schindler K, Leung H, Lehnertz K, Elger CE. How generalised are secondarily "generalised" tonic clonic seizures? J Neurol Neurosurg Psychiatry 78: 993-996, 2007.

18. Blumenfeld H, Varghese GI, Purcaro MJ, et al. Cortical and subcortical networks in human secondarily generalized tonic-clonic seizures. Brain 132: 999-1012, 2009.

19. Varghese GI, Purcaro MJ, Motelow JE, et al. Clinical use of ictal SPECT in secondarily generalized tonic-clonic seizures. Brain 132: 2102-2113, 2009.

The Internal Medicine is an Open Access journal distributed under the Creative Commons Attribution-NonCommercial-NoDerivatives 4.0 International License. To view the details of this license, please visit (https://creativecommons.org/licenses/ by-nc-nd/4.0/).

(C) 2019 The Japanese Society of Internal Medicine Intern Med 58: 1151-1155, 2019 